\title{
Motivação do diabético tipo 2 para o tratamento não farmacológico
}

\section{Motivation for a diabetic type 2 patient to adhere a non-pharmacological treatment}

\author{
Heva Lílian Bezerra Albuquerque do Santos ${ }^{1}$ \\ Fabíola Maria Ribeiro do Nascimento ${ }^{1}$ \\ Clara Maria Silvestre Monteiro de Freitas ${ }^{2}$ \\ Ana Elizabeth Vieira da Cunha ${ }^{2}$ \\ Denise Maria Martins Vancea ${ }^{2,3}$
}

\section{Resumo}

O presente estudo teve como objetivo verificar o que motiva o indivíduo diabético a frequentar um programa de exercício físico supervisionado. Esta investigação trata-se de uma pesquisa de cunho descritivo-quantiqualitativo de campo, em que foram avaliados 19 diabéticos do tipo2 (DM2); o instrumento utilizado foi um questionário validado para Escala de Motivação para o Tratamento (EMT) adaptada para a população diabética. A escala é constituída de 19 itens dos quais 13 avaliam a motivação intrínseca para o tratamento e 06 itens restantes avaliam a motivação extrínseca. Para análise estatística, utilizou-se o pacote SPSS 16.0 e os dados foram descritos em valores percentuais. Os motivos intrínsecos que apresentaram uma frequência elevada estavam relacionados com os fatores psicossociais $(84,2 \%)$ e de saúde e qualidade de vida $(73,7 \%)$, estes comprometem o ingresso e/ou permanência no tratamento do indivíduo DM2 na aderência ao tratamento. Ao mesmo tempo, os motivos extrínsecos que apresentaram maior frequência foram fatores interpessoais $(57,9 \%)$ o que demonstra o quanto é determinante o apoio das pessoas que fazem parte do convívio do DM2 no auxílio ao tratamento. Com isso, pôde-se concluir que a motivação intrínseca, especificamente nos aspectos cognitivos e de mudança de comportamento são fatores determinantes para aderência ao tratamento não farmacológico.

\section{Palavras-chave}

Motivação, Diabetes Mellitus e Exercício Físico.

\begin{abstract}
This study aimed to determine what motivates the diabetic to attend a supervised physical exercise program. This investigation is a search for a descriptive quanti-qualitative of field, which evaluated 19 diabetic single type 2 (DM2), the instrument was a validated questionnaire for Motivation Scale for Treatment (EMT) adapted to the diabetic population. The scale is constituted of 19 items, where 13 evaluate intrinsic motivation for treatment and the remaining 6 items measure extrinsic motivation for treatment. For statistical analysis was used SPSS 16.0 and the data was described in percentages. The intrinsic reasons that showed a high frequency are related to psychosocial factors (84.2\%) and health and quality of life (73.7\%), they undertake to enter and / or permanence of the DM2 in the treatment adherence. At the same time, the extrinsic reasons factors was shower more high frequency were the interpersonal factors (57.9\%) that demonstrates how crucial is the support of people who are part of the conviviality of T2DM in aid treatment. Consequently, we concluded that the intrinsic motivation, specifically in the cognitive aspects and behavioral change are key factors relevant to adherence to non-pharmacological treatment.
\end{abstract}

\section{Keywords}

Motivation, Type 2 Diabetes and Physical Exercise.
Rev Bras Ativ Fis Saúde p. 485-494 DOI: http://dx.doi.org/10.12820/2317$1634.2012 \mathrm{v} 17 \mathrm{n} 6 \mathrm{p} 485$

1 Universidade Gama Filho, Rio de Janeiro, RJ, Brasil.

2 Programa Exercício Supervisionado para Diabéticos (Doce Vida) e Grupo de Pesquisa em Estilo de Vida e Saúde (GPES), Curso de Educação Física da Escola Superior de Educação Física, Universidade de Pernambuco, Recife, PE, Brasil

3 UNIPECLIN - Grupo de Pesquisa Exercício Físico e Doenças Crônicas não Transmissiveis e do Grupo de Pesquisa, Escola Superior de Educação Física, Universidade de Pernambuco, Recife, PE, Brasil. 


\section{INTRODUÇÃO}

A Diabetes Mellitus, de acordo com as Diretrizes da Sociedade Brasileira de Diabetes $^{1}$ (2009), trata-se de uma síndrome caracterizada por distúrbios metabólicos que apresentam em comum a hiperglicemia, por uma deficiência na secreção ou ação do hormônio insulina. Suas principais formas são o diabetes mellitus tipo 1 (DM1), quando existe deficiência absoluta de insulina, e o diabetes mellitus tipo 2 (DM2), caracterizado por resistência à insulina com insuficiente elevação compensatória da secreção desse hormônio ${ }^{2}$.

O DM2 afeta vários domínios da qualidade de vida, incluindo saúde física, saúde psicológica, relacionamento social e pressão social, sendo necessária uma abordagem integral de cuidado que envolva esses elementos ${ }^{3,4}$.

A dificuldade apresentada no tratamento associa-se ao intensivo controle da glicemia, dos determinantes fisiológicos e psicossociais que contribuem para o desânimo do portador de DM2 ${ }^{5}$. A literatura tem indicado que o indivíduo com diabetes apresenta dificuldades para aderir a todo o programa terapêutico, consistindo em controle metabólico, dieta adequada, medicação e exercício físico ${ }^{3}$. A falta de motivação e a não adesão ao tratamento constitui uma das falhas do programa aumentando a morbimortalidade nessa população ${ }^{6}$.

Conviver com esta doença implica em adotar um estilo de vida saudável, incluindo a prática regular de exercício físico, sendo esta um fator de suma importância na conquista da qualidade de vida, pois proporciona sensação de conforto físico e mental, alívio, bem-estar, satisfação pessoal como afirma $\mathrm{Saba}^{7}$ (2001) em seu estudo. Por isso, os conceitos de promoção da saúde e bem-estar, nos quais o exercício físico atua positivamente são largamente difundidos ${ }^{8}$.

Os efeitos motivacionais do exercício físico nessa população são diversos, por meio da conquista de independência de drogas terapêuticas, redução do declínio funcional, ganhos de força, redução do peso e melhora da função cognitiva'.

Nessas dimensões, merece ser realçado o conceito de motivação para a prática de exercício físico. De acordo com Samulski ${ }^{10}$ (2002) e Machado ${ }^{11}$ (1995), a motivação pode ser entendida como um processo intencional, ativo e dirigido a uma meta a qual depende da relação de fatores pessoais (intrínsecos) e ambientais (extrínsecos).

Um estudo realizado por Chagas e Samulski ${ }^{12}$ (1992) em academias de ginástica destaca os seguintes aspectos motivadores que levam as pessoas a frequentá-las: manter a boa forma, melhorar o condicionamento físico, aumentar o bem estar social e psicológico, melhorar o estado de saúde e o prazer em realizar as atividades físicas. Esse processo não se torna diferente em indivíduos diabéticos, os motivos citados acima são compartilhados por essa população, porém pela diminuição da capacidade física e a morbidade que essa doença causa, os níveis de motivação são menores ${ }^{13}$ na maioria dos diabéticos.

O exercício físico regular é o tratamento de destaque para indivíduos com $\mathrm{DM} 2^{14}$. Nesta direção, o estudo tem como objetivo verificar o que motiva o indivíduo diabético a frequentar um programa de exercício físico supervisionado.

\section{MÉTODOS}

A presente investigação trata-se de uma pesquisa de cunho descritivo-quantiqualitativo de campo, devido ao grau de apreciação dirigido a ela. 


\section{Seleção da Amostra}

Foram avaliados 19 indivíduos com diabetes mellitus do tipo 2 (DM2), de ambos os gêneros, fisicamente ativos, recrutados do Doce Vida - Programa de Exercício Físico Supervisionado para Diabéticos da Escola Superior de Educação Física da Universidade de Pernambuco.

Para a pesquisa, foram adotados os seguintes critérios de inclusão: diagnóstico de DM2 e acordo com o THE EXPERT COMMITTEE ONTHE DIAGNOSIS AND CLASSIFICATION OF DIABETES MELLITUS ${ }^{15}$ (2000), ter mais de 50 anos de idade, ser praticante de exercício físico há pelo menos seis meses. E, como critérios de exclusão indivíduos pré-diabéticos, DM1 e com menos de seis meses de prática de exercício físico.

Durante o processo de coleta de dados, os sujeitos foram devidamente esclarecidos sobre os objetivos e procedimentos adotados nessa investigação, os quais assinaram o Termo de Consentimento Livre e Esclarecido de acordo com a resolução de número 196/96 que aprova as diretrizes e normas da pesquisa envolvendo seres humanos. Esta pesquisa teve aprovação pelo Comitê de Ética da Universidade de Pernambuco, protocolo no 060/11.

A amostra do presente estudo participarou de palestra sobre a importância do exercício físico no controle metabólico de indivíduos com diabetes tipo 2, na tentativa de apresentar o exercício físico como forma de intervenção terapêutica não farmacológica.

\section{Programa de Exercício Físico}

O programa de treinamento supervisionado teve duração de 18 meses, o que levou os avaliadores do presente estudo a estipular um tempo mínimo de 6 meses, visto que este é o menor tempo para promover aderência ao exercício físico ${ }^{16}$. O programa foi realizado nas dependências da Escola Superior de Educação Física/UPE, sob a supervisão de professores e acadêmicos de Educação Física. As sessões foram realizadas no período matutino, no ginásio de esportes e na sala de musculação, após a refeição de rotina.

Os sujeitos foram divididos em três modalidades de treinamento: grupo treinamento aeróbio (GTA), grupo treinamento resistido (GTR) e grupo treinamento combinado (GTC). Todos treinavam três vezes por semana, em dias alternados. $\mathrm{O}$ programa foi dividido em três momentos: 10 minutos de aquecimento, 40 minutos de treinamento e 10 minutos de volta à calma.

- GTA: Realizava 40 minutos de exercícios de caminhada a $60 \%$ da frequência cardíaca máxima (FC máx.) de acordo com o teste de esforço (protocolo de Bruce na esteira), progredindo para 80\% da FCmáx, utilizando para o treinamento a frequência cardíaca de reserva predita pelo ACSM ${ }^{17}$ (2009), localizado no quadro 1.

- GTR: Realizava 40 minutos de exercícios de musculação (cadeira extensora, rosca direta, cadeira flexora, tríceps coice, abdutora, remada, flexora e elevação lateral), a progressão da carga foi executada por meio da falha concêntrica momentânea dentro da margem de 10 a 16 repetições máximas ${ }^{16,17}$.

- GTC: Realizava 20 minutos de exercícios de musculação (idem GTR) e 20 minutos de caminhada (idem GTA).

\section{Instrumento e Técnica de Coleta}

O instrumento de coleta de dados foi um questionário devidamente validado para 
Escala de Motivação para o Tratamento (EMT) ${ }^{6}$ (APÊNDICE 1), adaptado pelos avaliadores para população diabética, tendo sido a mesma constituída por 19 itens, dos quais 13 avaliam a motivação intrínseca para o tratamento e os 06 itens restantes avaliam a motivação extrínseca. Convém ressaltar que foi utilizada a escala de Likert de 4 pontos para medir a motivação, dos quais 1 nada importante, 2 pouco importante, 3 importante e 4 muito importante. Os itens foram construídos considerando as dimensões relacionadas à saúde e à qualidade de vida, aspectos psicossociais e a relação interpessoal. As informações foram obtidas através de entrevista estruturada, com duração média de 30 minutos, na qual os entrevistados se limitaram a responder as perguntas contidas no instrumento e determinaram o grau de importância de cada motivo, de acordo com a escala adotada. Justifica-se essa atitude pelo fato de que os praticantes deviam ter o máximo de compreensão do delineamento do questionário, a fim de garantir uma maior fidedignidade. Participaram da coleta de dados 02 (dois) estagiários, treinados e coordenados pela equipe de pesquisa.

\section{Variáveis Dependentes e Independentes}

As variáveis dependentes deste estudo foram os aspectos motivacionais, relacionados à motivação intrínseca e extrínseca, acerca do tratamento não farmacológico em DM2 com o foco no exercício físico e para variável independente utilizou-se suporte familiar, escolaridade e renda familiar.

\section{Análise Estatística}

Os dados passaram por um tratamento estatístico, utilizando-se o pacote SPSS (Statistical Package for Social Sciences) 16.0 para Windows. Por meio desse software foram realizadas as estatísticas descritivas das variáveis dependentes as quais são apresentadas em valores percentuais ao longo do texto.

\section{RESULTADO}

As características gerais da amostra são apresentadas na Tabela 1.

Os motivos pelos quais os diabéticos participam de um programa de exercício físico foram analisados sob duas perspectivas: 1) os que se revelaram ser "muito importante" para a maioria da amostra e; 2) os que foram classificados como "nada importante" pela mesma.

Quadro 1 - Progressão do treinamento aeróbio de acordo com ACSM (2009).

\begin{tabular}{|lcccc|}
\hline Fase do Programa & Semana & $\begin{array}{c}\text { Frequência } \\
\text { do exercício } \\
\text { (sessões/sem.) }\end{array}$ & $\begin{array}{c}\text { Intensidade } \\
\text { de exercício } \\
\text { (\% FCR) }\end{array}$ & $\begin{array}{c}\text { Duração da } \\
\text { Sessão de } \\
\text { Exercício (min.) }\end{array}$ \\
\hline & 1 & 3 & $40-50$ & $15-20$ \\
\cline { 2 - 5 } & 2 & 3 & $40-50$ & $20-25$ \\
\hline Estágio Inicial & 3 & 3 & $50-60$ & $20-25$ \\
\cline { 2 - 5 } & 4 & 3 & $50-60$ & $25-30$ \\
\cline { 2 - 5 } & $5-7$ & 3 & $60-70$ & $30-40$ \\
\cline { 2 - 5 } & $11-10$ & 3 & $60-70$ & $30-40$ \\
\hline & $14-16$ & 3 & $60-80$ & $40-50$ \\
\hline & $17-20$ & 3 & $60-80$ & $50-60$ \\
\hline
\end{tabular}


Tabela 1 - Características gerais da amostra.

\begin{tabular}{lcccccc}
\hline & $\begin{array}{c}\text { Meninos } \\
(\mathrm{n}=6)\end{array}$ & Máx & Mín & $\begin{array}{c}\text { Meninas } \\
(\mathrm{n}=13)\end{array}$ & Máx & Mín \\
\hline Sexo (\%) & 31,6 & 0 & 0 & 68,4 & 0 & 0 \\
\hline Idade (anos) & 67,0 & 80 & 50 & 65,0 & 75 & 55 \\
\hline Tempo de diagnóstico (anos) & 17,0 & 30 & 1 & 6,0 & 30 & 1 \\
\hline Tempo de exercício (anos) & 2,0 & 10 & 1,0 & 1,0 & 12 & 1 \\
\hline Complicações crônicas secundáias & 2 & 3 & 1 & 1 & 2 & 1 \\
\hline Medicamento & 3,0 & 3 & 2 & 2,0 & 5 & 1 \\
\hline GTA & 0 & 0 & 0 & 3 & 3 & 0 \\
\hline GTR & 5 & 5 & 0 & 0 & 0 & 0 \\
\hline GTC & 2 & 2 & 0 & 2 & 2 & 0 \\
\hline
\end{tabular}

Os dados são apresentados em mediana e valores de máximo (máx) e mínimo (mín).

A tabela 2 representa os motivos intrínsecos que determinam a aderência ao tratamento por meio do controle glicêmico e da prática do exercício físico supervisionado. Os motivos intrínsecos que apresentaram maior constância foram os relacionados com os fatores psicossociais e de saúde e qualidade de vida.

Tabela 2 - Fatores determinantes na motivação intrínseca.

\begin{tabular}{|c|c|c|c|c|c|c|c|c|}
\hline & \multicolumn{2}{|c|}{ Nada importante } & \multicolumn{2}{|c|}{ Pouco importante } & \multicolumn{2}{|c|}{ Importante } & \multicolumn{2}{|c|}{ Muito importante } \\
\hline & $\mathrm{n}$ & $\%$ & $\mathrm{~N}$ & $\%$ & $\mathrm{n}$ & $\%$ & $\mathrm{n}$ & $\%$ \\
\hline${ }^{*}$ Fazê-lo, para mim, um desafio pessoal & 1 & 5,3 & 0 & 0,0 & 5 & 26,3 & 13 & 68,4 \\
\hline * *Acredito que ao fazê-lo vou melhorar & 0 & 0,0 & 0 & 0,0 & 5 & 5,3 & 14 & 73,7 \\
\hline *Me sentiria culpado se não fizesse o que o médico disse. & 1 & 5,3 & 3 & 15,8 & 5 & 26,3 & 10 & 52,6 \\
\hline${ }^{*}$ Me sentiria mal comigo mesmo se não o fizesse. & 0 & 0,0 & 2 & 10,5 & 6 & 31,6 & 11 & 57,9 \\
\hline $\begin{array}{l}\text { **É entusiástico manter a minha glicemia dentro dos } \\
\text { valores recomendados }\end{array}$ & 0 & 0,0 & 1 & 5,3 & 6 & 31,6 & 12 & 63,2 \\
\hline **Acredito que é importante para me manter saudável & 0 & 0,0 & 0 & 0,0 & 5 & 26,3 & 14 & 73,7 \\
\hline *Sentiria vergonha de mim mesma se não o fizesse. & 3 & 15,8 & 0 & 0,0 & 2 & 10,5 & 14 & 73,7 \\
\hline *É mais fácil fazê-lo do que ficar a pensar nisso. & 1 & 5,3 & 0 & 0,0 & 5 & 26,3 & 13 & 68,4 \\
\hline $\begin{array}{l}\text { *Pensei seriamente no assunto e acredito que é a } \\
\text { melhor coisa a fazer. }\end{array}$ & 0 & 0,0 & 0 & 0,0 & 4 & 21,1 & 15 & 79 \\
\hline *Sinto que são as melhores coisas que posso fazer por mim. & 1 & 5,3 & 1 & 5,3 & 3 & 15,8 & 14 & 73,7 \\
\hline${ }^{*}$ Me sentiria culpado se não o fizesse. & 2 & 10,5 & 0 & 0,0 & 3 & 15,8 & 14 & 73,7 \\
\hline *São as melhores escolhas que eu posso tomar. & 0 & 0,0 & 0 & 0,0 & 6 & 31,6 & 13 & 68,4 \\
\hline $\begin{array}{l}\text { *Se trata de um desafio aprender a viver com o meu } \\
\text { diabetes. }\end{array}$ & 1 & 5,3 & 0 & 0,0 & 2 & 10,5 & 16 & 84,2 \\
\hline
\end{tabular}

* Fatores psicossociais e ** Saúde e qualidade de vida

O fator psicossocial que obteve maior frequência foi "Se trata de um desafio aprender a viver com o meu diabetes" (84,2\%). Este demonstra o compromisso do indivíduo diabético tipo 2 (DM2) com o tratamento, sendo este considerado na escala como um fator "muito importante", por homens e mulheres.

Os fatores de saúde e qualidade de vida que obtiveram maior frequência foram "Acredito que ao fazê-lo vou melhorar" (73,7\%) e "Acredito que é importante para me manter saudável" (73,7\%), estes demonstram a preocupação com o estilo de vida, sendo esta uma dimensão considerada "muito importante", dentre os motivos intrínsecos de indivíduos DM2. 
A tabela 3 representa os motivos extrínsecos que determinam a aderência ao tratamento por meio do controle glicêmico e da prática do exercício físico supervisionado. Os fatores interpessoais apresentaram maior constância dentre os motivos extrínsecos.

Tabela 3 - Fatores determinantes na motivação extrínseca

\begin{tabular}{|c|c|c|c|c|c|c|c|c|}
\hline & \multicolumn{2}{|c|}{$\begin{array}{c}\text { Nada } \\
\text { importante }\end{array}$} & \multicolumn{2}{|c|}{$\begin{array}{c}\text { Pouco } \\
\text { importante }\end{array}$} & \multicolumn{2}{|c|}{ Importante } & \multicolumn{2}{|c|}{$\begin{array}{c}\text { Muito } \\
\text { importante }\end{array}$} \\
\hline & $\mathrm{n}$ & $\%$ & $\mathrm{n}$ & $\%$ & $\mathrm{n}$ & $\%$ & $\mathrm{n}$ & $\%$ \\
\hline ***As outras pessoas ficariam furiosas comigo se eu não fizesse & 5 & 26,3 & 1 & 5,3 & 2 & 10,5 & 11 & 57,9 \\
\hline *** Quero que o médico pense que sou um bom doente. & 5 & 26,3 & 2 & 10,5 & 9 & 47,4 & 3 & 15,8 \\
\hline ** *Não quero que as outras pessoas se decepcionem comigo. & 3 & 15,8 & 1 & 5,3 & 8 & 42,1 & 7 & 36,8 \\
\hline ** As outras pessoas ficariam aborrecidas comigo se eu não fizesse. & 5 & 26,3 & 5 & 26,3 & 6 & 31,6 & 3 & 15,8 \\
\hline *Quero que os outros vejam que sou capaz de fazê-lo. & 4 & 21,1 & 1 & 5,3 & 5 & 26,3 & 9 & 47,4 \\
\hline **O médico disse-me para fazê-lo. & 5 & 26,3 & 2 & 10,5 & 7 & 36,8 & 5 & 26,3 \\
\hline
\end{tabular}

*Fatores psicossociais; * Saúde e qualidade de vida e *** Relação Interpessoal.

O fator interpessoal considerado "muito importante" foi "As outras pessoas ficariam furiosas comigo se eu não fizesse" (57,9\%), este demonstra o quanto é determinante o apoio das pessoas que fazem parte do convívio do DM2 no auxílio ao tratamento.

\section{DISCUSSÃO}

Os resultados do presente estudo demonstram em geral, que indivíduos DM2 apresentam um elevado nível de motivação para o tratamento por meio do exercício físico, sendo que os motivos intrínsecos apresentaram maior freqüência do que os motivos extrínsecos.

Isso pode ser explicado pelos motivos intrínsecos corresponderem ao interesse do DM2 em conhecer, aprender e participar do tratamento ${ }^{18}$, pois este sentimento age em concordância com aquilo que o paciente espera, e isso advém de uma natureza multifatorial (escolaridade, renda familiar, acesso a informação de qualidade, suporte familiar... $)^{6}$.

Os motivos intrínsecos decorrentes de fatores psicossociais tornam-se menos resistentes à medida que os anos se passam ${ }^{19}$. Na seguinte frase: "Se trata de um desafio aprender a viver com o meu diabetes" (84,2\%), demonstra a incerteza dos perigos que podem ser alcançados por esta enfermidade, este sentimento contribui para a busca da manutenção e melhora do seu estado de saúde, por meio de estratégias de autocuidado como mudança nos hábitos alimentares, prática de exercício físico diário e o controle de redução de peso corporal ${ }^{20,21}$.

Ainda decorrente dos motivos intrínsecos, as frases: "Acredito que ao fazê-lo vou melhorar" (73,7\%) e "Acredito que é importante para me manter saudável" (73,7\%), estas dimensões foram consideradas "muito importantes". Isso pode ser explicado pelo que demonstrou o estudo de Oliveira ${ }^{22}$ (2011), que revelou a crença pelos diabéticos na utilização do exercício físico como estratégia para controle da glicemia nessa população ${ }^{22}$.

Os resultados do presente estudo corroboram com o de Apóstolo ${ }^{6}$ (2007), que investigou por meio de um questionário estruturado, a incerteza da doença e da motivação para o tratamento em DM2, demonstrando que o mais alto grau de motivação para o tratamento advém de motivos intrínsecos nos aspectos relativos à adoção de um estilo de vida saudável e à melhoria e manutenção do estado de saúde. 
Alguns autores acreditam "ainda" que a baixa adesão a um estilo de vida saudável, por meio de dietas e exercício físico, pode estar vinculada à forma como os profissionais orientam os diabéticos. Tendo em vista que a proposta geral para a alimentação do diabético não deve ser restritiva, impositiva e carregada de expressões proibitivas, e sim formada por um cardápio variado, balanceado, dentro de um limite preestabelecido ${ }^{23}$.

Em relação à prática do exercício físico, os benefícios à saúde para portadores de DM ocorrem mesmo quando esta se inicia tardiamente, contribuindo para a redução do peso corporal, diminuição do risco para o desenvolvimento de doenças cardiovasculares e doenças secundárias do diabetes, aumento na capacidade da função motora, além de benefícios nutricionais e melhora no padrão do sono ${ }^{24}$.

Segundo a Sociedade Brasileira de Diabetes ${ }^{1}$ (2009), a orientação nutricional e o estabelecimento de dieta para controle de pacientes com DM, incluindo o exercício físico, são considerados terapias de primeira escolha ${ }^{24}$.

No que se refere à motivação extrínseca, o presente estudo observou que o motivo extrínseco que apresentou maior constância estava relacionado aos fatores interpessoais. A frase: "As outras pessoas ficariam furiosas comigo se eu não fizesse" $(57,9 \%)$, demonstra o quanto é determinante o apoio das pessoas que fazem parte do convívio do DM2 no auxílio ao tratamento.

Alguns estudos verificaram que o suporte social familiar é um facilitador na adoção de condutas saudáveis, visto que a família é considerada como fonte de apoio para a pessoa com DM2, sendo importante a participação desta no processo educativo que favorecer a adoção de hábitos saudáveis, tanto da parte do paciente quanto de sua rede familiar, isto influenciar de forma positiva a adesão ao tratamento e controle glicêmico ${ }^{25,26}$.

Alguns estudos verificaram que o suporte social familiar é um facilitador na adoção de condutas saudáveis, visto que a família é considerada como fonte de apoio para o indivíduo com DM2, sendo importante a participação dela no processo educativo que favorece a adoção de hábitos saudáveis, tanto por parte do paciente quanto de sua rede familiar, isto pode influenciar de forma positiva a adesão ao tratamento e controle glicêmico ${ }^{19,27}$.

Não foi objetivo do presente estudo verificar a influência de cada modalidade de treinamento na variável motivação, mas como o exercício físico, na sua forma global, assistiria aos indivíduos DM2 motivando-os para o tratamento da doença. Outra limitação foi instrumento utilizado na coleta de dados, pois foi realizada uma entrevista em que os avaliadores não puderam interferir na pergunta ou entendimento da mesma, podendo desta forma haver distorções ou tendenciosidades por parte dos avaliados. Apesar disto verifica-se que o instrumento utilizado ofereceu dados relevantes e coerentes com aqueles encontrados na literatura da área.

\section{CONSIDERAÇÕES FINAIS}

No que concerne à motivação para o tratamento, os resultados obtidos revelam que a maioria dos pacientes DM2 apresenta alto grau de motivação para o tratamento. É todavia, na dimensão da motivação intrínseca especificamente nos aspectos relativos aos fatores psicossociais e de saúde e qualidade de vida que os pacientes em estudo evidenciam ter um elevado grau de motivação.

Relativamente aos aspectos onde os pacientes apresentam um grau de motivação mais baixo, estes se referem aos motivos que levam os pacientes com diabetes a comportar-se de acordo com o que os outros esperam deles, aspectos da moti- 
vação extrínseca. Estes resultados mostram-nos que os diabéticos tipo 2 parecem agir mais de acordo com as suas motivações intrínsecas do que com as motivações extrínsecas.

\section{Contribuiç̧ão dos autores}

As autoras Heva Lílian Bezerra Albuquerque dos Santos e Fabíola Maria Ribeiro do Nascimento participaram de as todas as etapas da pesquisa (coleta de dados, análise e o processo da escrita). As autoras, Clara Maria Silvestre Monteiro de Freitas, Ana Elizabeth Vieira da Cunha e Denise Maria Martins Vancea participaram da orientação e revisão final do artigo.

\section{Agradecimentos/Financiamento}

Gostaríamos de agradecer a todos aqueles que fazem parte do Doce Vida Programa de Exercício Físico Supervisionado para Diabéticos da Escola Superior de Educação Física da Universidade de Pernambuco.

Especialmente aos pacientes diabéticos que se disponibilizaram a responder ao questionário contribuindo para o nosso conhecimento.

Esse trabalho foi financiado pelos próprios pesquisadores.

\section{REFERÊNCIAS}

1. DIRETRIZES DA SOCIEDADE BRASILEIRA DE DIABETES / Sociedade Brasileira de Diabetes. - [3.ed.]. - Itapevi, SP: A. Araújo Silva Farmacêutica, 2009.

2. Franguas R, Soares SM, Bronstein M. Depressão e Diabetes Mellitus. Rev. Psiq. Clín. 2009; 36(3): 93-9.

3. Santos EC, Zanetti ML, Otero LM, Santos MA. O cuidado sob a ótica do paciente diabético e de seu principal cuidador. Rev. Latino-am Enferm. 2005; 13(3): 397-403.

4. Eren I, Erdi O, Sahin M. The effect of depression on quality of life of patients with type II diabetes mellitus. Depress Anxiety 2008; 25(2): 98-106.

5. Faro B. The effect of diabetes on adolescents' quality of life. Pediatr. Nurs 1999; 25(3):247-53, 286.

6. Apóstolo JLA, Viveiros CSC, Nunes HIR, Domingues HRF. Incerteza na doença e motivação para o tratamento em diabéticos tipo 2. Rev. Latino-am Enfermagem. Aug 2007;15(4): 575-82.

7. Saba FKF. Aderência à prática do exercício físico em academias. São Paulo: Manole; 2001.

8. Freitas C, Santiago M, Viana A, Leão A, Freyre C. Aspectos motivacionais que influenciam a adesão e manutenção de idosos a programa de exercícios físicos. Rev. Bras. Cineantropometria e Desempenho Humano 2007; 9(1): 92-100.

9. Willey KA, Sing MA. Battiing insulin reistance in elderly obese people whith type 2 diabetes: Bring on the heavy weights. Diabetes Care 2003; 26: 1580-1588.

10. Samulski, D. Psicologia do esporte. Manole: 1ed. 2002.

11. Machado AA. Importância da motivação para o movimento humano. IN: Perspectivas interdisciplinares em educação física, São Paulo: Soc. Bras. Des. Educação Física. 1995.

12. Chagas M, Samulski D. Análise da motivação para atividades físicas em academias de ginástica de Belo horizonte. Belo Horizonte UFMG (Projeto premiado de Iniciação Científica), 1992.

13. Fischer T, Skovlund SE. Dificuldades do dia a dia e o desgaste psicológico no diabetes tipo 1 e tipo 2 (EASD - European association for the Study of Diabetes, 35th Annual Meeting, Bruxelas. Diabetes Clínica 1999;3(5): 282-284.

14. Vancea DMM, Vancea JN, Pires MI et al. Effect of frequency of physical exercise on glycemic control and body composition in type 2 diabetic patients. Arq. Bras. Cardiol. 2009;92(1): 23-30.

15. THE EXPERT COMMITTEE ON THE DIAGNOSIS AND CLASSIFICATION OF DIABETES MELLITUS. Report of the expert committee on the diagnosis and classification of diabetes mellitus. Diabetes Care 2000;23 (supp.1): 4-19.

16. Fleck SJ, Kreamer WJ. Fundamentos do treinamento de força muscular. Tradução Jerri Luiz Ribeiro. - 3, ed. - Porto Alegre: Artmed, 2006. 
17. AMERICAN COLLEGE OF SPORTS MEDDICINE (ACSM). Position stand on Progression models in resistance training for healthy adults. Medicine and Science in Sports and Exercise 2009; 41(3): 687-708.

18. Ryan RM, Deci EL. Self-determination theory and facilitation of intrinsic motivation, social development and weel-being. American Psychologist 2000; 55(1):68-78.

19. Péres DS, Franco LJ, Santos MA. Sentimentos de mulheres após o diagnóstico de diabetes tipo 2. Rev Latino-am Enfermagem, jan-fev 2008; 16(1). Disponível em<http//www.eerp.usp.br/ rlae>. Acesso: 20 de agosto.

20. Ogden J. Psicologia da saúde. Lisboa: Climepsi; 1999.

21. Grillo MFF. Caracterização e práticas de autocuidado de pessoas com diabete melito tipo 2 de uma unidade básica de saúde. Dissertação (Mestrado em Enfermagem) - Universidade Federal do Rio Grande do Sul. Escola de enfermagem. Curso de Mestrado em enfermagem, 2005. Disponível em: http://www.lume.ufrgs.br/bitstream/handle/ 10183/6967/000493094. pdf Acesso em 02 de novembro de 2012.

22. Oliveira NF, Souza e Melo CB, Zanetti ML, Santo MA. Diabetes Mellitus: desafios relacionados ao autocuidado abordado em Grupo de Apoio Psicológico. Rev. Bras. Enferm, Brasília, mar-abr 2011; 64(2): 301-7.

23. Mahan LK, Escott-Stump S. Krause - alimentos, nutrição e dietoterapia.10a ed. São Paulo: Roca; 2002

24. Silva LMC, Pallha PF, Barbosa GR, Protti ST, Ramos AS. Aposentados com diabetes tipo 2 na Saúde da Família em Ribeirão Preto, São Paulo - Brasil. Rev. Esc. Enferm. 2010; 44: 462-8.

25. Thoolen B, Ridder D, Bensing J, Gorter K, Rutten G. Who participates in Diabetes selfmanagement interventions? Issues of recruitment and retainment. Diabetes Educator 2007; 33(3):465-74.

26. Zanetti ML, Biagg MV, Santos MA, Péres DS, Teixeira CRS. O cuidado à pessoa diabética e as repercussões na família. Rev Bras Enferm 2008; 61(2): 186-92.

27. Blanski CRK, Lenardt MH. A Compreensão da Terapêutica Medicamentosa pelo Idoso. Rev Gaúcha de Enferm. 2005;26:180-8.

$$
\begin{array}{r}
\text { Endereço para Correspondência } \\
\text { Heva Lílian Bezerra Albuquerque dos Santos } \\
\text { Escola Superior de Educação Física - ESEF } \\
\text { Rua Arnóbio Marques, 310, } \\
\text { Santo Amaro, Recife-PE. } \\
\text { CEP: } 50.100-130 \\
\text { (81) } 31833375 / 8569-0020 \\
\text { Email: hevasantos@hotmail.com }
\end{array}
$$




\section{APÊNDICE 1}

\section{Escala de Motivação para o Tratamento (EMT)}

(0) nada importante; (1) pouco importante; (2) importante; (3) muito importante.

\section{Itens da Motivação para o tratamento}

A) Eu faço o meu tratamento para o diabetes e/ou controlo a minha glicemia por que...

2 - As outras pessoas ficariam furiosas comigo se eu não fizesse. （ ）

3 - Fazê-lo é, para mim, um desafio pessoal. ( )

4 - Acredito que ao fazê-lo vou melhorar a minha saúde. ( )

5 - Me sentiria culpado se não fizesse o que o médico disse. ( )

6 - Quero que o médico pense que sou um bom doente. ( )

7 - Me sentiria mal comigo mesmo se não o fizesse. ( )

8 - É entusiástico manter a minha glicemia dentro dos valores recomendados.

9 - Não quero que as outras pessoas se decepcionem comigo.

B) A razão pela qual cuido do meu diabetes e faço regularmente exercício físico, é por que...

10 - As outras pessoas ficariam aborrecidas comigo se eu não fizesse. ( )

11 - Acredito que é importante para me manter saudável. （ )

12 - Sentiria vergonha de mim mesma se não o fizesse. （ )

13 - É mais fácil fazê-lo do que ficar a pensar nisso. （ ）

14 - Pensei seriamente no assunto e acredito que é a melhor coisa a fazer.

15 - Quero que os outros vejam que sou capaz de fazê-lo.

16 - O médico disse-me para fazê-lo.

17 - Sinto que são as melhores coisas que posso fazer por mim. （ ）

18 - Me sentiria culpado se não o fizesse.

19 - São as melhores escolhas que eu posso tomar.

20 - Se trata de um desafio aprender a viver com o meu diabetes. 